\title{
Synthesis of Titania-Silica Materials by Sol-Gel
}

\author{
Rubia F. S. Lenza, Wander L. Vasconcelos* \\ Laboratory of Ceramic Materials - LMC \\ Department of Metallurgical and Materials Eng. - UFMG \\ Rua Espírito Santo, 35 - 2o andar, Centro, Belo Horizonte - MG, Brazil
}

Received: November 21, 2000; Revised: September 23, 2002

\begin{abstract}
In this work $\mathrm{TiO}_{2}-\mathrm{SiO}_{2}$ glasses containing as much as $20 \mathrm{~mol} \%$ of $\mathrm{TiO}_{2}$ were prepared via solgel process using titanium and silicon alkoxides, in the presence of chlorine, in the form of titanium tetrachloride or $\mathrm{HCl}$. The gels were heat-treated until $800{ }^{\circ} \mathrm{C}$. X-ray diffraction and Fourier transform infrared spectroscopy were used to understand the structural properties of $\mathrm{TiO}_{2}-\mathrm{SiO}_{2}$ oxides calcined at different temperatures and to evaluate the homogeneity of these materials. The degree of the compactness of the silica network is inferred from the frequency of the asymmetric stretching vibrations of Si-O-Si bonds. Formation of Si-O-Ti bridges, as monitored by the intensity of characteristic $945 \mathrm{~cm}^{-1}-960 \mathrm{~cm}^{-1}$ vibration, is particularly prominent if the method of basic two-step prehydrolysis of silicon alkoxide, addition of titanium alkoxide and completion of hydrolysis was used.
\end{abstract}

Keywords: $\mathrm{TiO}_{2}-\mathrm{SiO}_{2}$, sol-gel, synthesis

\section{Introduction}

Sol-gel is a low temperature method that produces ceramics and glasses with better purity and homogeneity than high temperature conventional process. Sol-gel has produced a wide range of compositions in several forms, such as powders, fibers, coatings, monoliths and porous membranes. The most attractive features of the sol-gel process include the production of compositions not possible with conventional ceramic preparation methods, along with the retention of the mixing level of the solution in the final product, often on the molecular scale ${ }^{1-5}$.

The sol-gel process involves the hydrolysis (1) and condensation (2) e (3) of metal alkoxides ${ }^{4,6,7}$ :

$$
\begin{aligned}
& \mathrm{M}-\mathrm{OR}+\mathrm{HOH} \rightarrow \mathrm{M}-\mathrm{OH}+\mathrm{ROH} \\
& \mathrm{M}-\mathrm{OH}+\mathrm{RO}-\mathrm{M} \rightarrow \mathrm{M}-\mathrm{O}-\mathrm{M}+\mathrm{ROH} \\
& \mathrm{M}-\mathrm{OH}+\mathrm{HO}-\mathrm{M} \rightarrow \mathrm{M}-\mathrm{O}-\mathrm{M}+\mathrm{HOH}
\end{aligned}
$$

By controlling the synthesis conditions carefully, these reactions may lead to a variety of structures, and to different final states for the materials ${ }^{5,6}$.

The incorporation of $\mathrm{TiO}_{2}$ into silica glasses has an important effect on the properties of the final glass. The ther- mal stability of $\mathrm{SiO}_{2}$ is high, but the chemical stability at high temperature in the presence of water is poor, resulting in the formation of surface hydroxyls ${ }^{7}$. Incorporation of $\mathrm{TiO}_{2}$ has proven to improve the chemical stability. In addition, a small molar percentage of $\mathrm{TiO}_{2}$ can be used to prepare silica glasses with almost zero thermal expansion coefficient due to the ability of $\mathrm{Ti}$ ions to coordenate tetrahedrally ${ }^{8}$.

Although the physical properties of the titania-silica glasses such as the thermal expansion coefficients, FTIR and Raman spectra are well documented, processing methods required to achieve and maintain an atomic distribution of titanium have been little investigated until recently. Conventional melt technology is limited for these glass compositions by the high separation of titania-rich phase at elevated temperature ${ }^{9,10}$.

Recently, sol-gel process has been investigated for synthesis of $\mathrm{TiO}_{2}-\mathrm{SiO}_{2}$ glasses ${ }^{5,-12}$. There still are problems, however, when the sol-gel processes are applied to binary systems ${ }^{11,13,14}$. The reactivities of titanium alkoxides are considerably higher than for silicon alkoxides. Therefore, care has to be taken in the synthesis of binary materials of this system to avoid homo-condensation ${ }^{7}$. The degree of homogeneity is dependent of processing parameters, such as the nature and chemical reactivities of reactants, the concentration of water and sequence of addition, the $\mathrm{pH}$ of reaction 
medium and the time and temperature of reactions ${ }^{13}$.

Heterogeneity due to different hydrolysis rates can be avoided in several ways. A commonly used strategy is the use of prehydrolysis process: the slowest reacting alkoxide is prehydrolysed prior to the addition of the second, fast reacting, alkoxide. The idea is that the newly added alkoxide will condense with partially hydrolysed sites on the polymeric species formed by the preceding hydrolysis steps ${ }^{5,7,13}$. Another approach consists on the control of hydrolysis rates of highly reactive alkoxide by using modified alkoxides, in which the added ligant is kinetically and/or thermodynamically more stable ${ }^{11,13}$. Another mean of controlling homogeneity is achieving a very low hydrolysis rate by restricting the availability of water or prehydrolysis of the slowerreacting component with a later addition of the faster hydrolysing component, and further addition of water to complete the hydrolysis. It is expected ${ }^{5}$ that this method should be more suitable for producing solids that are mixed on a molecular scale.

In the present study, we focus on the influence of some preparation variables on the resulting structure of $\mathrm{TiO}_{2}-\mathrm{SiO}_{2}$ mixed oxides that can be used as ceramic membranes ${ }^{7}$ and as support in catalytic systems ${ }^{5}$. In the mixed oxides, the weight fraction of $\mathrm{TiO}_{2}$ was held constant at $20 \mathrm{~mol} \%$. The preparation variables being tested include, hydrolysis $\mathrm{pH}$, the sequence of hydrolysis and mixing, the drying temperature and the influence of polymerisation control reagent.

\section{Experimental}

Tetraethyl orthosilicate (TEOS, Aldrich Co.) and titanium isopropoxide (TIP, Aldrich Co.) were used as precursors. Alcohol was used as solvent: ethanol (Merck Co.) and isopropanol (Química Moderna Co.). Hydrochloric acid (Merck Co.) and acetic acid (Merck Co.) were used as catalysts. As polymerisation control reagent we used $\mathrm{TiCl}_{4}$ (Merck Co.). Binary sols are abbreviated as STX, where X is the method used in synthesis:

(A) Two-step acid hydrolysis, where TEOS was hydrolysed with the addition of deionised water, ethanol and hydrochloric acid. The water/alkoxide molar ratio used was $R=2$. After stirring for 15 minutes, a mixture of TIP, isopropanol and acetic acid was added. The $\mathrm{pH}$ of the solution was adjusted with hydrochloric acid to $\mathrm{pH}$ of 1 . A second addition of water (enough to reach water/alkoxide molar ratio $\mathrm{R}=4$ ) was dropwised. $\mathrm{A}$ clear yellow solution was cast into cylindrical containers and gelled at $40{ }^{\circ} \mathrm{C}$.

(B) Two-step basic hydrolysis, where the acid catalyst $\mathrm{HCl}$, used in the method $\mathrm{A}$, was replaced by a solution of $\mathrm{NaOH}$. The solution was adjusted for $\mathrm{pH}$ of 9.0 and precipitation occurred spontaneously to pro- duce a powder dispersion resembling milk. Following precipitation, colloid compacts were produced by evaporation of the solvent under constant agitation. The dispersion was cast into cylindrical containers and gelled at $40{ }^{\circ} \mathrm{C}$.

(C) Separate prehydrolysis followed by mixing of sols. TEOS was prehydrolysed in alcoholic solution of $\mathrm{pH}$ 1.0 and $\mathrm{R}=4$. TIP was prehydrolysed in alcohol medium (isopropanol) with $\mathrm{pH}$ of 3.0 (acetic acid) and $\mathrm{R}=4$. The sols mixed under vigorous stirring for $30 \mathrm{~min}$. The clear yellow solution was cast and gelled at $40{ }^{\circ} \mathrm{C}$.

(D) Hydrolysis of TEOS and TIP with $\mathrm{TiCl}_{4}$ as polymerisation control reagent. The hydrolysis of binary metal alkoxides was carried out by addition, under vigorous stirring, of the mixture of TEOS and TIP in a mixture of alcohol (ethanol) and water in which the alkoxide mixture contained titanium tetrachloride as chlorine source $(\mathrm{Cl} / \mathrm{Ti}$ molar ratio of 1.2$)$. The final $\mathrm{pH}$ was adjusted for 0.5 with addition of $\mathrm{HCl}$. The water/alkoxide molar ratio used was $\mathrm{R}=4$. The yellow solution was cast and gelled at $40{ }^{\circ} \mathrm{C}$.

The gels were aged at $60{ }^{\circ} \mathrm{C}$ and dried at $110{ }^{\circ} \mathrm{C}$. After drying, the gels were grounded to white fine powders and heated at $600^{\circ} \mathrm{C}$ and $800{ }^{\circ} \mathrm{C}$, maintaining the samples $6 \mathrm{~h}$ at each temperature.

The crystallisation behaviour of the samples was examined by X-ray diffractometry (XRD Philips Co.) using $\mathrm{Cu}$ Ka radiation. Diffuse reflectance FTIR (DRFTIR) spectra were recorded in a FTIR Paragon-1000 of Perkin Elmer Co. using diffuse reflectance accessory of Perkin-Elmer Co. Spectra were collected with a $4 \mathrm{~cm}^{-1}$ resolution and 200 scans have been accumulated for each spectrum in the frequency range of $4400 \mathrm{~cm}^{-1}$ to $400 \mathrm{~cm}^{-1}$. Samples were grounded and diluted to $5 \% \mathrm{w} / \mathrm{w}$ with $\mathrm{KBr}$ salt (Merck, Co.) and were allowed to cool to room temperature in air before recording spectra. In order to compare peaks intensities, we used the same mass of each sample to obtain DRFTIR spectra.

\section{Results and Discussion}

\section{STA}

The DRFTIR spectra of STA samples at $110^{\circ} \mathrm{C}, 600^{\circ} \mathrm{C}$ and $800{ }^{\circ} \mathrm{C}$ are presented in Fig. 1 . The spectrum at $110^{\circ} \mathrm{C}$ is characterised by a dominant band at $1030 \mathrm{~cm}^{-1}$ with a shoulder at $1160 \mathrm{~cm}^{-1}$, assigned to asymmetric stretching vibrations, $\mathrm{ASTO}_{1}$ and $\mathrm{ASLO}_{1}$ modes respectively, of the tetrahedral $\mathrm{SiO}_{4}$ co-ordination unit. The corresponding symmetric motion $\left(\mathrm{STO}_{1}\right)$ is detected at $797 \mathrm{~cm}^{1}{ }^{5,9}$. We observed a band around $980 \mathrm{~cm}^{-1}$ which is assigned as stretching vibration of silanol groups, $\mathrm{Si}-\mathrm{OH}$, owing to non-bridg- 


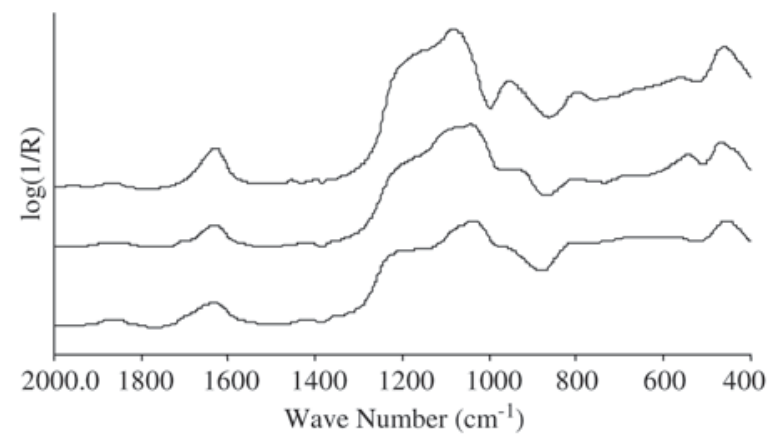

Figure 1. DRFTIR spectra of STA samples treated at $110{ }^{\circ} \mathrm{C}$, $600{ }^{\circ} \mathrm{C}$ and $800{ }^{\circ} \mathrm{C}$ (top to bottom).

ing oxygen associated to proton ${ }^{5,9,14}$ and a minor feature around $950 \mathrm{~cm}^{-1}$, which can be associated with vibrations of Si-O-Ti bridges ${ }^{5,11}$. The peak occurring at $465 \mathrm{~cm}^{-1}$ results from rocking vibration of Si-O-Si bonds ${ }^{15}$. The spectral features of $\mathrm{TiO}_{2}$ can be discerned at around $540 \mathrm{~cm}^{-1}$ and $440 \mathrm{~cm}^{-1}$. Drying this sample at $600{ }^{\circ} \mathrm{C}$ and $800{ }^{\circ} \mathrm{C}$ shifts the ASTO frequency to $1032 \mathrm{~cm}^{-1}$ and $1074 \mathrm{~cm}^{-1}$ (with a shoulder at $1030 \mathrm{~cm}^{1}$ ), respectively. The corresponding $\mathrm{ASLO}_{1}$ vibration mode frequencies are $1170 \mathrm{~cm}^{-1}$ and $1190 \mathrm{~cm}^{-1}$, respectively. Calcination at high temperatures results in a considerable decrease of the $980 \mathrm{~cm}^{1}$ band intensity, as compared with the corresponding gel dried at $110{ }^{\circ} \mathrm{C}$. The weak band centred around $950 \mathrm{~cm}^{1}$, which shifted to a high frequency of $952 \mathrm{~cm}^{-1}$ at $800{ }^{\circ} \mathrm{C}$, did not change its intensity. The characteristic peaks of titania $\left(540 \mathrm{~cm}^{-1}\right.$ and $\left.440 \mathrm{~cm}^{-1}\right)$ decreased in intensity. The X-ray diffraction spectra show the presence of crystallites of anatase and rutile, as shown in Fig. 2. These results indicate that the acid two-step prehydrolysis process promotes the occurrence of low proportion of homo-condensation reactions. The STA samples calcinated at different temperatures present titania separated domains.

\section{$S T B$}

DRFTIR spectra of mixed oxide samples prepared by a basic prehydrolysis of TEOS, addition of TIP, completion of hydrolysis, and calcination at $110^{\circ} \mathrm{C}, 600^{\circ} \mathrm{C}$ and $800^{\circ} \mathrm{C}$ are presented in Fig. 3. The spectrum of gel treated at $110^{\circ} \mathrm{C}$ exhibit one prominent difference from the others. A broad band between $1500 \mathrm{~cm}^{-1}$ and $1400 \mathrm{~cm}^{-1}$, which is characteristic of organic groups ${ }^{15}$, is present. This is probably a consequence of basic hydrolysis of TEOS, which leads to an incomplete hydrolysis of alkoxide ${ }^{16}$. This band disappears at high temperatures. The $\mathrm{ASTO}_{1}$ frequencies for gels dried at different temperatures are higher than for samples STA. At $110{ }^{\circ} \mathrm{C} \mathrm{ASTO}_{1}$ band is centred around $1075 \mathrm{~cm}^{-1}$ with a

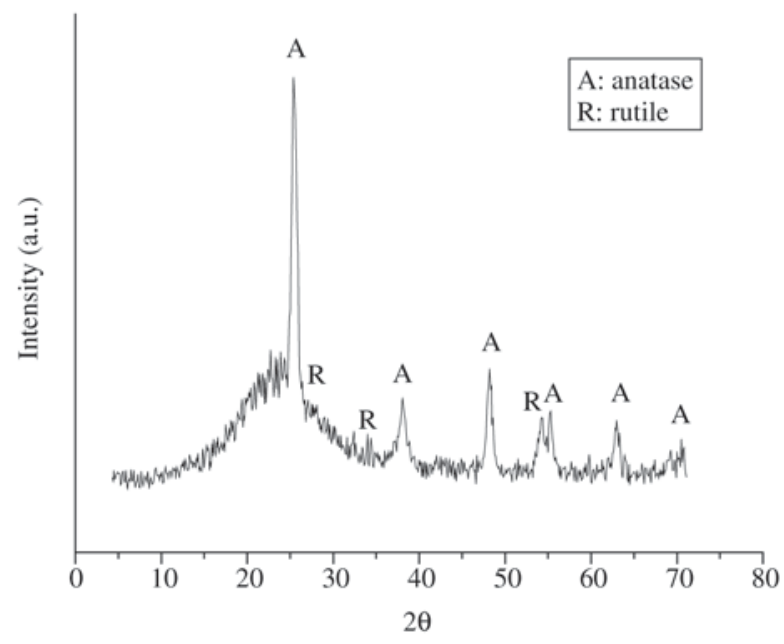

Figure 2. X ray diffraction peaks to STA sample.

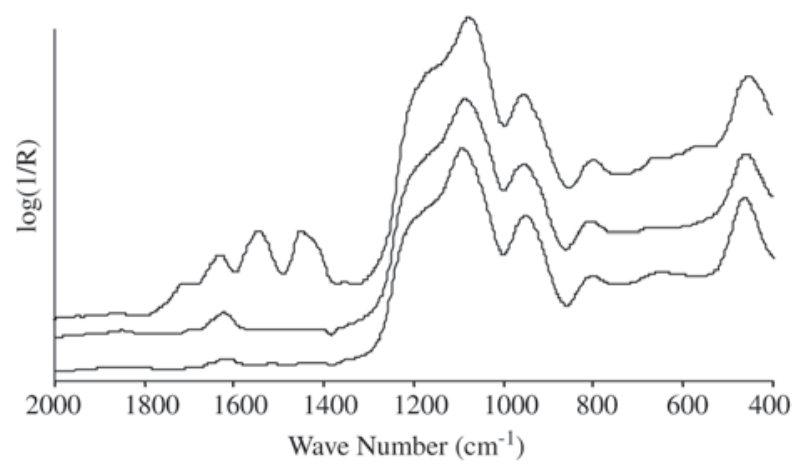

Figure 3. DRFTIR spectra of STB samples treated at $110^{\circ} \mathrm{C}$, $600{ }^{\circ} \mathrm{C}$ and $800{ }^{\circ} \mathrm{C}$ (top to bottom).

shoulder at $1170 \mathrm{~cm}^{-1}\left(\mathrm{ASLO}_{1}\right)$. At $600{ }^{\circ} \mathrm{C}$ the frequency of this band shifts to $1080 \mathrm{~cm}^{-1}$, with a shoulder at $1170 \mathrm{~cm}^{-1}$ and at $800^{\circ} \mathrm{C}$ the frequency shifts to $1191 \mathrm{~cm}^{-1}$ with a shoulder at $1180 \mathrm{~cm}^{-1}$. The $\mathrm{STO}_{1}$ vibration band appears at $796 \mathrm{~cm}^{-1}$ at $110^{\circ} \mathrm{C}$ and shifts to a high frequencies of 800 $\mathrm{cm}^{-1}$ with calcination. The band characteristic of Si-O-Ti bonds at around $950 \mathrm{~cm}^{-1}-955 \mathrm{~cm}^{-1}$ appears highly more intense than in sample STA and its intensity increase with temperature, indicating that the samples prepared by method $\mathrm{B}$ present more Si-O-Ti bonds. The rocking vibration band of silica network did not present any change with temperature. Interestingly, in the spectra of STB samples no spectral features of titania can be discerned. This is an indication that the samples prepared by method B did not present $\mathrm{TiO}_{2}$ domains. No diffraction lines were observed in the $\mathrm{X}$-ray spectra, only a broad peak characteristic of an amor- 
phous silicate can be discerned, as can be seen in Fig. 4 . These results indicate that the method $\mathrm{B}$ allows the preparation of homogeneous $\mathrm{SiO}_{2}-\mathrm{TiO}_{2}$ glasses.

STC

Diffuse reflectance FTIR spectra of mixed oxides prepared by complete hydrolysis of the components before mixing are presented in Fig. 5. The spectra of STC sample dried at different temperatures are characterised by ASTO $_{1}$ frequencies at $1070 \mathrm{~cm}^{-1}\left(110{ }^{\circ} \mathrm{C}\right), 1082 \mathrm{~cm}^{-1}\left(600^{\circ} \mathrm{C}\right)$, and $1086 \mathrm{~cm}^{-1}\left(800{ }^{\circ} \mathrm{C}\right)$. The $\mathrm{ASLO}_{1}$ frequencies also shift to higher frequencies with the increasing of temperature. The $\mathrm{STO}_{1}$ band vibration appears at $794 \mathrm{~cm}^{-1}$ at $110{ }^{\circ} \mathrm{C}$ and its frequency shifts to a high frequency of $800 \mathrm{~cm}^{-1}$ with calcination. The intensity of $\mathrm{SiOH}$ absorption band decreases with the increasing of temperature. The Si-O-Ti band appears at $950 \mathrm{~cm}^{-1}$ (weak) and its intensity also decreases

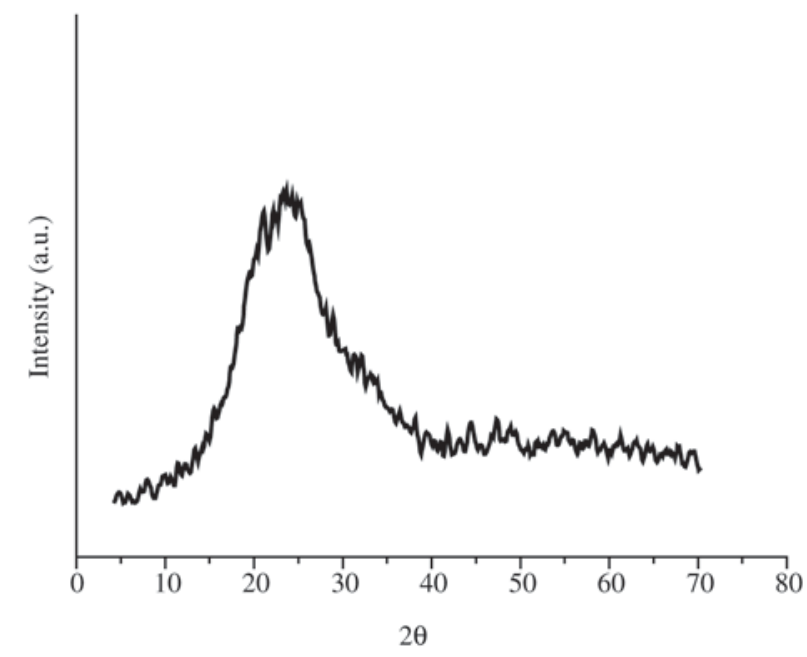

Figure 4. X ray diffraction peaks to STB sample.

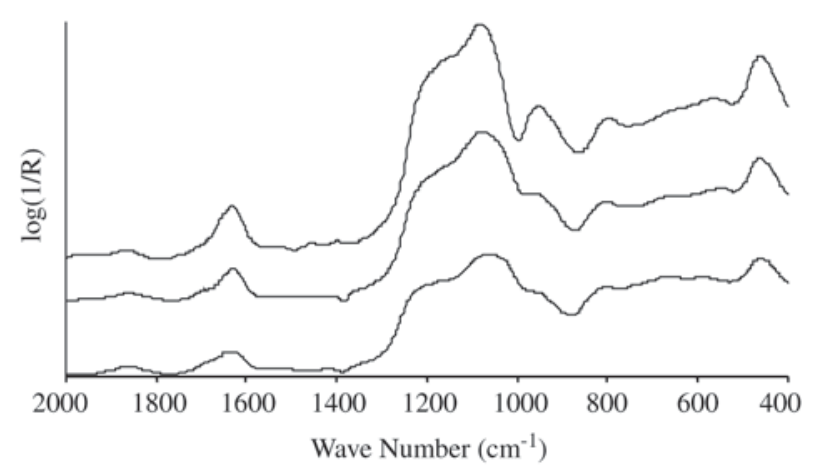

Figure 5. DRFTIR spectra of STC samples treated at $110^{\circ} \mathrm{C}$, $600{ }^{\circ} \mathrm{C}$ and $800{ }^{\circ} \mathrm{C}$ (top to bottom). upon heating. The band due to titania network appears at $560 \mathrm{~cm}^{-1}$. These spectra denote that samples obtained by method $\mathrm{C}$ present titania domains with low proportion of Si-O-Ti bonds. The X-ray diffraction spectrum, shown in Fig. 6, denotes the presence of crystallisation of titania (anatase and rutile). Thus, we conclude that the method of complete hydrolysis of the two alkoxides before mixing is not adequate for preparing homogeneous binary glasses.

STD

The DRFTIR spectra of mixed oxide $\mathrm{SiO}_{2}$ and $\mathrm{TiO}_{2}$ prepared using $\mathrm{TiCl}_{4}$ as polymerisation control reagent are shown in Fig. 7. The ASTO frequency of the sample treated at $110{ }^{\circ} \mathrm{C}$ appears at $1075 \mathrm{~cm}^{-1}$ and shifts to $1086 \mathrm{~cm}^{-1}$ at $800{ }^{\circ} \mathrm{C}$. The $\mathrm{ASLO}_{1}$ and $\mathrm{STO}_{1}$ band frequencies also shift to higher frequencies with the increasing of temperature. The intensity of silanol band decreases with calcination. The

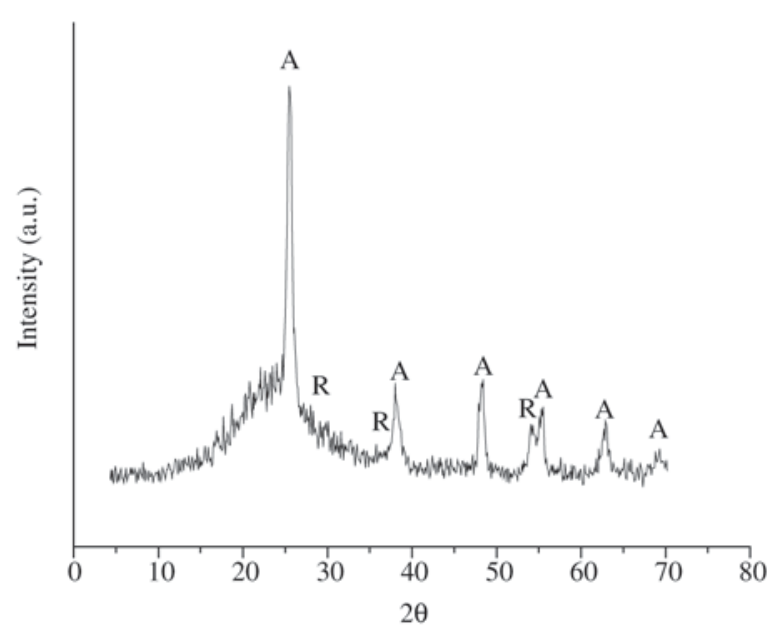

Figure 6. X ray diffraction peaks to STC sample.

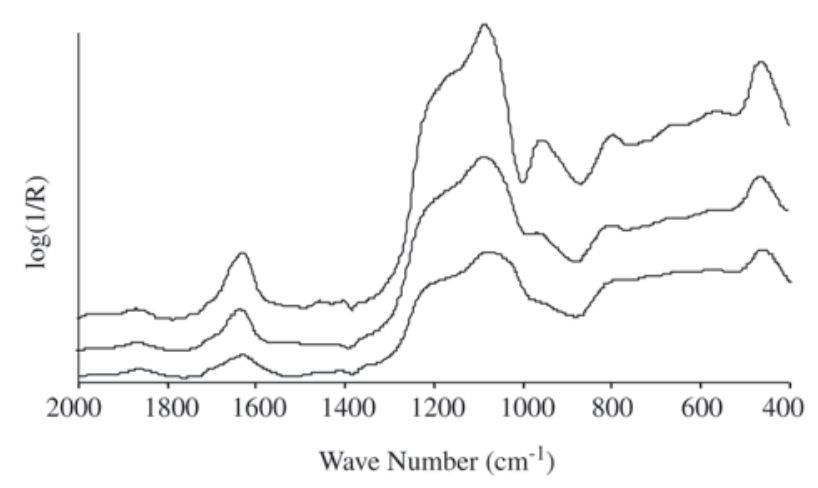

Figure 7. DRFTIR spectra of STD samples treated at $110{ }^{\circ} \mathrm{C}$, $600{ }^{\circ} \mathrm{C}$ and $800{ }^{\circ} \mathrm{C}$ (top to bottom). 
characteristic band of Si-O-Ti bridges appears around $950 \mathrm{~cm}^{-1}$ and its intensity stays roughly constant with the increasing of temperature. The titania characteristic bands $\left(560 \mathrm{~cm}^{-1}\right.$ and $440 \mathrm{~cm}^{-1}$ ) are present for all temperatures studied. The X-ray diffraction spectrum of the STD sample is presented in Fig. 8. This spectrum shows the titania crystallisation in anatase phase. We noted that samples obtained by method $\mathrm{D}$ and dried at $800{ }^{\circ} \mathrm{C}$ present a heterogeneous network with separated oxides and a low amount of Si-O-Ti bridges.

We would expect that $\mathrm{TiCl}_{4}$ could avoid the homo-condensation reactions by the exchange reaction with titanium isoproxide ${ }^{11}$ :

$$
\mathrm{TiCl}_{4}+3 \mathrm{Ti}\left(\mathrm{OPr}^{\mathrm{i}}\right)_{4} \rightarrow 4 \mathrm{Ti}\left(\mathrm{Pr}^{\mathrm{i}}\right)_{3} \mathrm{Cl}
$$

Although the replacement of alkoxy groups by chlorine decreases the degree of association of titanium alkoxide, which could prevent preferential segregation of titanium and silicon oxides, the result indicates the occurrence of homocondensation reactions. Thus, we can conclude that a large amount of chlorine is required to avoid homo-condensation reactions.

The positions of the maxima $\mathrm{ASTO}_{1}$ vibration mode of $\mathrm{Si}-\mathrm{O}-\mathrm{Si}$ bonds, which may serve as indication of the degree of cross-linking of the silica network, are highest in the spectra of the gels prepared by the two-step basic prehydrolysis of silicon alkoxide method. This is expected since the basic medium promotes high rates of polymerisation and consequently, a more compact oxide network.

An ideal $\mathrm{TiO}_{2}-\mathrm{SiO}_{2}$ gel would consist only of $\mathrm{Si}-\mathrm{O}-\mathrm{Si}$ and Si-O-Ti bonds, which can be detected by means of FTIR. If the gel were not homogeneous, crystallisation of $\mathrm{TiO}_{2}$ would occur and be detected by XRD. The band in the

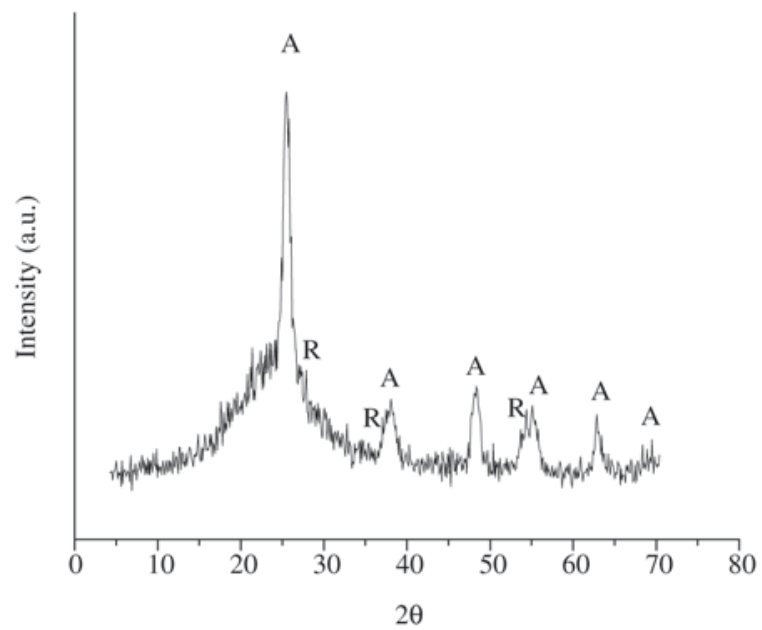

Figure 8. X-ray diffraction peaks to STD sample.
$945 \mathrm{~cm}^{-1}-960 \mathrm{~cm}^{-1}$ range is typical for $\mathrm{TiO}_{2}-\mathrm{SiO}_{2}$ glasses or crystalline titanium-silicate ${ }^{5,9}$. It has been assigned to a local impurity mode of a $\mathrm{SiO}_{4}$ structural unit bonded to $\mathrm{Ti}^{\mathrm{IV}}$ (i.e., the stretching modes of $\mathrm{Si}-\mathrm{O}-\mathrm{Ti}$ bridges). The relative intensity of this characteristic absorption can be used to evaluate the amount of $\mathrm{Ti}^{\mathrm{IV}}$ cations incorporated into the silica network in tetrahedral co-ordination ${ }^{5,9}$. It can be noted that only STB samples present this band with high intensity, indicating that method B promotes the formation of a high proportion of hetero-condensation reactions. In addition, the lack of features due to titania in the spectra of STB samples indicates absence of $\mathrm{TiO}_{2}$ domains. The X-ray diffraction spectra confirm these observations: only in the spectra of STB samples no diffraction lines were observed.

\section{Conclusion}

Sol-gel derived glasses in the $\mathrm{TiO}_{2}-\mathrm{SiO}_{2}$ system, obtained through methods $\mathrm{A}, \mathrm{C}$ and $\mathrm{D}$, are heterogeneous in terms of $\mathrm{TiO}_{2}$ distribution. As far as cross-linking of the silica network is concerned, hydrolysis in basic and/or high temperature drying promote the formation of strongly cross-linked networks.

An intense signal due to $\mathrm{Si}-\mathrm{O}-\mathrm{Ti}$ bridges, the lack of features of $\mathrm{TiO}_{2}$ domains on the DRFTIR spectra and X-ray diffraction spectra attest that method $\mathrm{B}$ is adequate to preparing homogeneous $\mathrm{TiO}_{2}-\mathrm{SiO}_{2}$ glasses with a high content of $\mathrm{TiO}_{2}$ and a highly compact oxide network.

\section{Acknowledgement}

The authors thank the financial support of CAPES, CNPQ and FAPEMIG.

\section{References}

1. Alkoxysilane - Http://www.b72.com/objects/stone/ axhome.html - 16/10/98.

2. Sham, E.L.; Fárfan-Torres, E.M.; Michel, R.L.; Gottifredi, J.C. Latin America Applied Research, v. 27, p. 245, 1997.

3. Hu, L.; Yoko, T.; Kozuka, H.; Sakka, S. Thin Solid Films, v. 219, p. 18, 1992.

4. Lenza, R.F.S.; Vasconcelos, W.L. Journal of Non-Crystalline Solids, v. 263, n. 1-3, p.164, 2000.

5. Schraml-Marth, M.; Walther, K.L.; Wokan, A.; Handy, B.E.; Baiker, A. Journal of Non-Crystalline Solids, v. 143, p. 93, 1992.

6. Brunet, F.; Cabane, B. Journal of Non-Crystalline Solids, v. 163, p. 211, 1993.

7. deLange, R.S.A.; Hehhink, J.H.A.; Kelzer, K.; Burggraaf, A.J. Journal of Non-Crystalline Solids, v. 191, p. 1, 1995.

8. Salvado, J.M.M.; Margaça, F.M.A.; Teixeira, J. Journal of Non-Crystalline Solids, v. 163, p. 115, 1993.

9. Minehan, W.T.; Messing, G.L.; Pantano, C.G. Journal of 
Non-Crystalline Solids, v. 108, p. 163, 1989.

10. Deng, Z.; Breval, E.; Pantano, C.G. Journal of NonCrystalline Solids, v. 100, p. 365, 1988.

11. Aizawa, M.; Nosaka, Y.; Fujii, N. Journal of Non-Crystalline Solids, v. 168, p. 49, 1994.

12. Martucci, A.; Schell, J.; Battaglin, G.; Guglielmi, M. Journal of Applied Research, v. 27, p. 245, 1997.

13. Pereira, M.M.; Clarck, A.E.; Hench, L.L. Journal of
Materials Synthesis and Processing, v. 2, n. 3, 1994.

14. Orgaz, F.; Rawson, H. Journal of Non-Crystalline Solids, v.82, p.378, 1986.

15. Lenza, R.F.S.; Vasconcelos, W.L. Materials Research, v. 4, p. 189, 2001.

16. Brinker, C.J.; Scherer, G.W. Sol-Gel Science: The Physics and Chemistry of Sol-Gel Processing, Academic Press, inc. San Diego, CA. P. 907, 1990. 